\title{
Optimal harvesting control and dynamics of two-species stochastic model with delays
}

\author{
Lidan Liu ${ }^{1}$ and Xinzhu Meng ${ }^{1,2^{*}}$
}

\begin{tabular}{l}
\hline${ }^{*}$ Correspondence: \\
mxz721106@sdust.edu.cn \\
${ }^{1}$ College of Mathematics and \\
Systems Science, Shandong \\
University of Science and \\
Technology, Qingdao, 266590, \\
P.R. China \\
2State Key Laboratory of Mining \\
Disaster Prevention and Control \\
Co-founded by Shandong Province \\
and the Ministry of Science and \\
Technology, Shandong University of \\
Science and Technology, Qingdao, \\
266590, P.R. China
\end{tabular}

266590, P.R. China

\begin{abstract}
Taking the stochastic effects on growth rate and harvesting effort into account, we propose a stochastic delay model of species in two habitats. The main aim of this paper is to investigate optimal harvesting and dynamics of the stochastic delay model. By using the stochastic analysis theory and differential inequality technology, we firstly obtain sufficient conditions for persistence in the mean and extinction. Furthermore, the optimal harvesting effort and the maximum of expectation of sustainable yield (ESY) are gained by using Hessian matrix, the ergodic method, and optimal harvesting theory of differential equations. To illustrate the performance of the theoretical results, we present a series of numerical simulations of these cases with respect to different noise disturbance coefficients.
\end{abstract}

Keywords: stochastic delay model; extinction; persistence in the mean; optimal harvesting policy

\section{Introduction}

The population dynamics could be affected by the process of migration among patches. Due to natural conditions, such as the geology, climate, and hydrology, and the human factors, which include the development of tourism and the locations of industries, the animal habitats have been divided into some small patches. In recent years, many scholars studied the persistence and extinction of species with diffusion [1-6]. The result of [7] shows that the stability of periodic solution has some connection with the diffusion coefficients. The the references mentioned, the models with diffusion affecting the growth rate were studied, whereas Allen [8] proposed a logistic model with diffusion that affects density dependence. It can be described by the following formulation:

$$
\frac{\mathrm{d} x_{j}(t)}{\mathrm{d} t}=x_{j}(t)\left[r_{j}-b_{j} x_{j}\right]+\sum_{k=1, k \neq j}^{m} D_{j k} x_{j}(t)\left[x_{k}(t)-\alpha_{j k} x_{j}(t)\right], \quad j=1, \ldots, m,
$$

where $x_{j}(j=1, \ldots, m)$ denotes the density of species $x$ in patch $j, r_{j}>0$ is the growth rate of $x_{j}, b_{j}>0$ stands for the density-dependent factor in patch $j, D_{j k} \geq 0$ represent the diffusion coefficient from patch $k$ to patch $j$, and $\alpha_{j k} \geq 0, j, k=1, \ldots, m$, are the diffusion boundary conditions.

It is quite a common phenomenon that the species are always affected by environmental fluctuations. The population growth usually suffers from the disturbance of ecological en-

(c) The Author(s) 2017. This article is distributed under the terms of the Creative Commons Attribution 4.0 International License (http://creativecommons.org/licenses/by/4.0/), which permits unrestricted use, distribution, and reproduction in any medium, provided you give appropriate credit to the original author(s) and the source, provide a link to the Creative Commons license, and indicate if changes were made. 
vironment, such as supply of food, climate change, and natural enemies, and the stochasticity can be described by white noise. The stochastic model has attracted the attention of many researchers, and more studies can be found in [9-18]. The growth rates affected by noises can be described by

$$
r_{i} \rightarrow r_{i}+\sigma_{i 1} \dot{B}_{i 1}(t), \quad i=1,2,
$$

where $\sigma_{i 1}$ is the intensity of $B_{i 1}(t)$.

To develop and manage the biological resource, it is important to consider the problem of optimal harvesting. If natural resources management is reasonable, then it can increase sustainable production and equitable profit. Bioeconomic models allow ecological resources or certain benefits to be suitably exploited. A number of researchers have paid particular attention to the study of optimal harvesting policy, and a lot of results have been obtained [19-25]. However, sometimes the harvesting may also be affected by humancaused disturbance, such as the price of labor power, crude oil, and goods, which could also be described as white noise. The proportion coefficient of harvesting can be replaced by

$$
E_{i} \rightarrow E_{i}+\sigma_{i 2} \dot{B}_{i 2}(t), \quad i=1,2,
$$

where $\sigma_{i 2}$ is the intensity of $B_{i 2}(t)$, and $B_{11}(t), B_{12}(t), B_{21}(t)$, and $B_{22}(t)$ are independent standard Brownian motions.

In model (1), we consider the stochastic effects not only on growth rate but also on harvesting effort to investigate the optimal harvesting problem of the following stochastic model:

$$
\left\{\begin{aligned}
\mathrm{d} x_{1}(t)= & x_{1}(t)\left[r_{1}-E_{1}-b_{1} x_{1}(t)\right] \mathrm{d} t+D_{1} x_{1}(t)\left[\mathrm{e}^{-d_{2} \tau_{2}} x_{2}\left(t-\tau_{2}\right)-\alpha_{1} x_{1}(t)\right] \mathrm{d} t \\
& +\sigma_{11} x_{1}(t) \mathrm{d} B_{11}(t)-\sigma_{12} x_{1}(t) \mathrm{d} B_{12}(t), \\
\mathrm{d} x_{2}(t)= & x_{2}(t)\left[r_{2}-E_{2}-b_{2} x_{2}(t)\right] \mathrm{d} t+D_{2} x_{2}(t)\left[\mathrm{e}^{-d_{1} \tau_{1}} x_{1}\left(t-\tau_{1}\right)-\alpha_{2} x_{2}(t)\right] \mathrm{d} t \\
& +\sigma_{21} x_{2}(t) \mathrm{d} B_{21}(t)-\sigma_{22} x_{2}(t) \mathrm{d} B_{22}(t),
\end{aligned}\right.
$$

where $E_{i} \geq 0(i=1,2)$ and $d_{i}(i=1,2)$ respectively denote harvesting effort and death rate of the species $x_{i}, \tau_{i} \geq 0(i=1,2)$ is the time delay caused by the diffusion of the species $x_{i}(i=1,2), D_{1}$ is the diffusion coefficient from patch 2 to patch 1 , whereas $D_{2}$ represents the diffusion coefficient from patch 1 to patch 2 , and $\alpha_{i}(i=1,2)$ represent the boundary conditions.

\section{Stochastic persistence and extinction}

For convenience, we define the following notations:

$$
\begin{aligned}
& \tau=\max \left\{\tau_{1}, \tau_{2}\right\}, \quad\langle f(t)\rangle=t^{-1} \int_{0}^{t} f(s) \mathrm{d} s, \\
& \langle f\rangle^{*}=\limsup _{t \rightarrow+\infty} t^{-1} \int_{0}^{t} f(s) \mathrm{d} s, \quad\langle f\rangle_{*}=\liminf _{t \rightarrow+\infty} t^{-1} \int_{0}^{t} f(s) \mathrm{d} s, \\
& M_{0}=\left(b_{1}+\alpha_{1} D_{1}\right)\left(b_{2}+\alpha_{2} D_{2}\right), \quad M=\left(b_{1}+\alpha_{1} D_{1}\right)\left(b_{2}+\alpha_{2} D_{2}\right)-D_{1} D_{2} \mathrm{e}^{-\left(d_{1} \tau_{1}+d_{2} \tau_{2}\right)},
\end{aligned}
$$




$$
M_{1}=k_{1}\left(b_{2}+\alpha_{2} D_{2}\right)+k_{2} D_{1} \mathrm{e}^{-d_{2} \tau_{2}}, \quad M_{2}=k_{1} D_{2} \mathrm{e}^{-d_{1} \tau_{1}}+k_{2}\left(b_{1}+\alpha_{1} D_{1}\right),
$$

where $k_{1}=r_{1}-E_{1}-0.5 \sigma_{11}^{2}-0.5 \sigma_{12}^{2}$ and $k_{2}=r_{2}-E_{2}-0.5 \sigma_{21}^{2}-0.5 \sigma_{22}^{2}$.

To begin with, we introduce some lemmas.

Lemma 2.1 ([21]) Suppose that $X(t) \in C\left(\Omega \times[0,+\infty), R_{+}\right)$.

(i) If there exist two positive constants $T$ and $\eta_{0}$ such that

$$
\ln X(t) \leq \eta t-\eta_{0} \int_{0}^{t} X(s) \mathrm{d} s+\sum_{j=1}^{m} \sigma_{j} W_{j}(t)
$$

for all $t \geq T$, where $\sigma_{j}, j=1, \ldots, m$, are constants, then

$$
\begin{cases}\langle X(t)\rangle^{*} \leq \eta / \eta_{0} & \text { a.s. if } \eta \geq 0 \\ \lim _{t \rightarrow+\infty} X(t)=0 & \text { a.s. if } \eta<0\end{cases}
$$

(ii) If there exist three positive constants $T, \eta$, and $\eta_{0}$ such that

$$
\ln X(t) \geq \eta t-\eta_{0} \int_{0}^{t} X(s) \mathrm{d} s+\sum_{j=1}^{m} \sigma_{j} W_{j}(t)
$$

for all $t \geq T$, then $\langle X(t)\rangle_{*} \geq \eta / \eta_{0}$ a.s.

Lemma 2.2 For any given initial value $x_{0}(t) \in C\left([-\tau, 0], R_{+}^{2}\right)$, there exists a function $x(t)$ that is the unique solution for model (4) on $t \geq-\tau$ and remains in $R_{+}^{2}$ with probability 1 .

The proof is similar to that in [17] and it is omitted here.

In order to obtain the properties of model (4), let us first analyze the following auxiliary system:

$$
\left\{\begin{aligned}
\mathrm{d} y_{1}(t)= & y_{1}(t)\left[r_{1}-E_{1}-b_{1} y_{1}(t)\right] \mathrm{d} t-\alpha_{1} D_{1} y_{1}^{2}(t) \mathrm{d} t \\
& +\sigma_{11} y_{1}(t) \mathrm{d} B_{11}(t)-\sigma_{12} y_{1}(t) \mathrm{d} B_{12}(t), \\
\mathrm{d} y_{2}(t)= & y_{2}(t)\left[r_{2}-E_{2}-b_{2} y_{2}(t)\right] \mathrm{d} t+D_{2} y_{2}(t)\left[\mathrm{e}^{-d_{1} \tau_{1}} y_{1}\left(t-\tau_{1}\right)-\alpha_{2} y_{2}(t)\right] \mathrm{d} t \\
& +\sigma_{21} y_{2}(t) \mathrm{d} B_{21}(t)-\sigma_{22} y_{2}(t) \mathrm{d} B_{22}(t) .
\end{aligned}\right.
$$

Lemma 2.3 If $k_{1}=r_{1}-E_{1}-0.5 \sigma_{11}^{2}-0.5 \sigma_{12}^{2}>0$, then any solution $y(t)=\left(y_{1}(t), y_{2}(t)\right)$ of model (5) satisfies

$$
\lim _{t \rightarrow+\infty}\left\langle y_{1}\right\rangle=\frac{k_{1}}{b_{1}+\alpha_{1} D_{1}} \quad \text { a.s. and } \begin{cases}\lim _{t \rightarrow+\infty} y_{2}(t)=0 & \text { a.s. if } M_{2}<0 \\ \lim _{t \rightarrow+\infty}\left\langle y_{2}\right\rangle=\frac{M_{2}}{M_{0}} & \text { a.s. if } M_{2}>0\end{cases}
$$

Proof Applying Itô's formula to system (5) leads to

$$
\begin{aligned}
\mathrm{d}\left(\ln y_{1}(t)\right)= & {\left[k_{1}-b_{1} y_{1}(t)-\alpha_{1} D_{1} y_{1}(t)\right] \mathrm{d} t+\sigma_{11} \mathrm{~d} B_{11}(t)-\sigma_{12} \mathrm{~d} B_{12}(t) } \\
\mathrm{d}\left(\ln y_{2}(t)\right)= & {\left[k_{2}-b_{2} y_{2}(t)+D_{2} \mathrm{e}^{-d_{1} \tau_{1}} y_{1}\left(t-\tau_{1}\right)-\alpha_{2} D_{2} y_{2}(t)\right] \mathrm{d} t } \\
& +\sigma_{21} \mathrm{~d} B_{21}(t)-\sigma_{22} \mathrm{~d} B_{22}(t)
\end{aligned}
$$


Integrating both sides of these two differential equations, we find

$$
\begin{aligned}
\ln y_{1}(t)-\ln y_{1}(0)= & k_{1} t-\left(b_{1}+\alpha_{1} D_{1}\right) \int_{0}^{t} y_{1}(s) \mathrm{d} s+\sigma_{11} B_{11}(t)-\sigma_{12} B_{12}(t), \\
\ln y_{2}(t)-\ln y_{2}(0)= & k_{2} t+D_{2} \mathrm{e}^{-d_{1} \tau_{1}} \int_{0}^{t} y_{1}\left(s-\tau_{1}\right) \mathrm{d} s \\
& -\left(b_{2}+\alpha_{2} D_{2}\right) \int_{0}^{t} y_{2}(s) \mathrm{d} s+\sigma_{21} B_{21}(t)-\sigma_{22} B_{22}(t) .
\end{aligned}
$$

Dividing both sides of (7) and (8) by $t$, we get

$$
\begin{aligned}
t^{-1} \ln \frac{y_{1}(t)}{y_{1}(0)}= & k_{1}-\left(b_{1}+\alpha_{1} D_{1}\right) t^{-1} \int_{0}^{t} y_{1}(s) \mathrm{d} s+t^{-1} \sigma_{11} B_{11}(t)-t^{-1} \sigma_{12} B_{12}(t) \\
t^{-1} \ln \frac{y_{2}(t)}{y_{2}(0)}= & k_{2}+D_{2} \mathrm{e}^{-d_{1} \tau_{1}} t^{-1} \int_{0}^{t} y_{1}\left(s-\tau_{1}\right) \mathrm{d} s-\left(b_{2}+\alpha_{2} D_{2}\right) t^{-1} \int_{0}^{t} y_{2}(s) \mathrm{d} s \\
& +t^{-1} \sigma_{21} B_{21}(t)-t^{-1} \sigma_{22} B_{22}(t) \\
= & k_{2}+D_{2} \mathrm{e}^{-d_{1} \tau_{1}} t^{-1} \int_{0}^{t} y_{1}(s) \mathrm{d} s+D_{2} \mathrm{e}^{-d_{1} \tau_{1}} t^{-1}\left[\int_{-\tau_{1}}^{0} y_{1}(s) \mathrm{d} s-\int_{t-\tau_{1}}^{t} y_{1}(s) \mathrm{d} s\right] \\
& -\left(b_{2}+\alpha_{2} D_{2}\right) t^{-1} \int_{0}^{t} y_{2}(s) \mathrm{d} s+t^{-1} \sigma_{21} B_{21}(t)-t^{-1} \sigma_{22} B_{22}(t) .
\end{aligned}
$$

By Lemma 2.1 we derive from (7) that $\left\langle y_{1}\right\rangle^{*} \leq \frac{k_{1}}{\left(b_{1}+\alpha_{1} D_{1}\right)}$ and $\left\langle y_{1}\right\rangle_{*} \geq \frac{k_{1}}{\left(b_{1}+\alpha_{1} D_{1}\right)}$. Hence, we have

$$
\lim _{t \rightarrow+\infty}\left\langle y_{1}\right\rangle=\frac{k_{1}}{b_{1}+\alpha_{1} D_{1}} \quad \text { a.s. }
$$

Using $\lim _{t \rightarrow+\infty} t^{-1} B_{1 j}(t)=0, j=1,2$, and substituting (11) into (9) yield

$$
\lim _{t \rightarrow+\infty} t^{-1} \ln y_{1}(t)=0 \quad \text { a.s. }
$$

Computing $(9) \times D_{2} \mathrm{e}^{-d_{1} \tau_{1}}+(10) \times\left(b_{1}+\alpha_{1} D_{1}\right)$ leads to

$$
\begin{aligned}
\left(b_{1}+\right. & \left.\alpha_{1} D_{1}\right) t^{-1} \ln \frac{y_{2}(t)}{y_{2}(0)}+D_{2} \mathrm{e}^{-d_{1} \tau_{1}} t^{-1} \ln \frac{y_{1}(t)}{y_{1}(0)} \\
= & M_{2}-M_{0}\left\langle y_{2}\right\rangle+\left(b_{1}+\alpha_{1} D_{1}\right) D_{2} \mathrm{e}^{-d_{1} \tau_{1}} t^{-1}\left[\int_{-\tau_{1}}^{0} y_{1}(s) \mathrm{d} s-\int_{t-\tau_{1}}^{t} y_{1}(s) \mathrm{d} s\right] \\
& +t^{-1}\left[D_{2} \mathrm{e}^{-d_{1} \tau_{1}}\left(\sigma_{11} B_{11}(t)-\sigma_{12} B_{12}(t)\right)+\left(b_{1}+\alpha_{1} D_{1}\right)\left(\sigma_{21} B_{21}(t) \sigma_{22} B_{22}(t)\right)\right] .
\end{aligned}
$$

From (11) we get

$$
\lim _{t \rightarrow+\infty} t^{-1} \int_{t-\tau_{1}}^{t} y_{1}(s) \mathrm{d} s=\lim _{t \rightarrow+\infty} t^{-1}\left[\int_{0}^{t} y_{1}(s) \mathrm{d} s-\int_{0}^{t-\tau_{1}} y_{1}(s) \mathrm{d} s\right]=0 \quad \text { a.s. }
$$

We can find from (12), (13), and (14) that, when $M_{2}<0, \lim _{t \rightarrow+\infty} y_{2}(t)=0$ a.s., and when $M_{2}>0, \lim _{t \rightarrow+\infty}\left\langle y_{2}\right\rangle=\frac{M_{2}}{M_{0}}$ a.s. 
Similarly, we give another auxiliary system to help us obtain the main results:

$$
\left\{\begin{aligned}
\mathrm{d} z_{1}(t)= & z_{1}(t)\left[r_{1}-E_{1}-b_{1} z_{1}(t)\right] \mathrm{d} t+D_{1} z_{1}(t)\left[\mathrm{e}^{-d_{2} \tau_{2}} z_{2}\left(t-\tau_{2}\right)-\alpha_{1} z_{1}(t)\right] \mathrm{d} t \\
& +\sigma_{11} z_{1}(t) \mathrm{d} B_{11}(t)-\sigma_{12} z_{1}(t) \mathrm{d} B_{12}(t) \\
\mathrm{d} z_{2}(t)= & z_{2}(t)\left[r_{2}-E_{2}-b_{2} z_{2}(t)\right] \mathrm{d} t-\alpha_{2} D_{2} z_{2}^{2}(t) \mathrm{d} t \\
& +\sigma_{21} z_{2}(t) \mathrm{d} B_{21}(t)-\sigma_{22} z_{2}(t) \mathrm{d} B_{22}(t)
\end{aligned}\right.
$$

Similarly to the proof of Lemma 2.3, we get the following:

Lemma 2.4 If $k_{2}=r_{1}-E_{1}-0.5 \sigma_{21}^{2}-0.5 \sigma_{22}^{2}>0$, then any solution $z(t)=\left(z_{1}(t), z_{2}(t)\right)$ of system (15) satisfies

$$
\left\{\begin{array}{ll}
\lim _{t \rightarrow+\infty} z_{1}(t)=0 & \text { a.s. if } M_{1}<0, \\
\lim _{t \rightarrow+\infty}\left\langle z_{1}\right\rangle=\frac{M_{1}}{M_{0}} & \text { a.s. if } M_{1}>0,
\end{array} \text { and } \quad \lim _{t \rightarrow+\infty}\left\langle z_{2}\right\rangle=\frac{k_{2}}{b_{2}+\alpha_{2} D_{2}} \quad\right. \text { a.s. }
$$

Moreover, we can derive the following equations:

$$
\lim _{t \rightarrow+\infty} t^{-1} \ln z_{2}(t)=0 \quad \text { a.s. }
$$

and

$$
\lim _{t \rightarrow+\infty} t^{-1} \int_{t-\tau_{2}}^{t} z_{2}(s) \mathrm{d} s=\lim _{t \rightarrow+\infty} t^{-1}\left[\int_{0}^{t} z_{2}(s) \mathrm{d} s-\int_{0}^{t-\tau_{2}} z_{2}(s) \mathrm{d} s\right]=0 \quad \text { a.s. }
$$

From Lemmas 2.1-2.4 we can obtain the following theorem.

Theorem 2.1 Suppose that $M>0$. The solution $x(t)=\left(x_{1}(t), x_{2}(t)\right)$ of system (4) has the following global asymptotic properties:

(i) If $k_{1}<0$ and $k_{2}<0$, then both $x_{1}$ and $x_{2}$ go to extinction almost surely (a.s.), that is,

$$
\lim _{t \rightarrow+\infty} x_{i}(t)=0 \quad \text { a.s., } i=1,2 .
$$

(ii) If $k_{1}>0$ and $M_{2}<0$, then $x_{1}$ is persistent in mean a.s., that is,

$$
\lim _{t \rightarrow+\infty}\left\langle x_{1}\right\rangle=\frac{k_{1}}{b_{1}+\alpha_{1} D_{1}} \quad \text { a.s. }
$$

and $x_{2}$ goes to extinction a.s.

(iii) If $k_{2}>0$ and $M_{1}<0$, then $x_{1}$ goes to extinction a.s., and $x_{2}$ is persistent in mean a.s., that is,

$$
\lim _{t \rightarrow+\infty}\left\langle x_{2}\right\rangle=\frac{k_{2}}{b_{2}+\alpha_{2} D_{2}} \quad \text { a.s. }
$$

(iv) If $M_{1}>0$ and $M_{2}>0$, then both $x_{1}$ and $x_{2}$ are persistent in mean a.s., that is,

$$
\lim _{t \rightarrow+\infty}\left\langle x_{1}\right\rangle=\frac{M_{1}}{M}, \quad \lim _{t \rightarrow+\infty}\left\langle x_{2}\right\rangle=\frac{M_{2}}{M} \quad \text { a.s. }
$$


Proof By stochastic comparison theorem we get

$$
x_{1}(t) \leq y_{1}(t), \quad x_{2}(t) \leq y_{2}(t)
$$

From (12) and (14) we can observe that

$$
\lim _{t \rightarrow+\infty} t^{-1} \ln x_{1}(t)=0
$$

and

$$
\lim _{t \rightarrow+\infty} t^{-1} \int_{t-\tau_{1}}^{t} x_{1}(s) \mathrm{d} s=0 .
$$

Similarly, we can also obtain

$$
x_{1}(t) \leq z_{1}(t), \quad x_{2}(t) \leq z_{2}(t)
$$

Then it follows from (17) and (18) that

$$
\lim _{t \rightarrow+\infty} t^{-1} \ln x_{2}(t)=0
$$

and

$$
\lim _{t \rightarrow+\infty} t^{-1} \int_{t-\tau_{2}}^{t} x_{2}(s) \mathrm{d} s=0
$$

Applying Itô's formula to system (4) yields

$$
\begin{aligned}
& \mathrm{d}\left(\ln x_{1}(t)\right)=\left[k_{1}-\left(b_{1}+\alpha_{1} D_{1}\right) x_{1}(t)+D_{1} \mathrm{e}^{-d_{2} \tau_{2}} x_{2}\left(t-\tau_{2}\right)\right] \mathrm{d} t+\sigma_{11} \mathrm{~d} B_{11}(t)-\sigma_{12} \mathrm{~d} B_{12}(t), \\
& \mathrm{d}\left(\ln x_{2}(t)\right)=\left[k_{2}-\left(b_{2}+\alpha_{2} D_{2}\right) x_{2}(t)+D_{2} \mathrm{e}^{-d_{1} \tau_{1}} x_{1}\left(t-\tau_{1}\right)\right] \mathrm{d} t+\sigma_{21} \mathrm{~d} B_{21}(t)-\sigma_{22} \mathrm{~d} B_{22}(t) .
\end{aligned}
$$

Integrating both sides of these two differential equations, we get

$$
\begin{aligned}
\ln \frac{x_{1}(t)}{x_{1}(0)}= & k_{1} t-\left(b_{1}+\alpha_{1} D_{1}\right) \int_{0}^{t} x_{1}(s) \mathrm{d} s+D_{1} \mathrm{e}^{-d_{2} \tau_{2}} \int_{0}^{t} x_{2}\left(s-\tau_{2}\right) \mathrm{d} s \\
& +\sigma_{11} B_{11}(t)-\sigma_{12} B_{12}(t) \\
= & k_{1} t-\left(b_{1}+\alpha_{1} D_{1}\right) \int_{0}^{t} x_{1}(s) \mathrm{d} s+D_{1} \mathrm{e}^{-d_{2} \tau_{2}} \int_{0}^{t} x_{2}(s) \mathrm{d} s \\
& +D_{1} \mathrm{e}^{-d_{2} \tau_{2}}\left[\int_{-\tau_{2}}^{0} x_{2}(s) \mathrm{d} s-\int_{t-\tau_{2}}^{t} x_{2}(s) \mathrm{d} s\right]+\sigma_{11} B_{11}(t)-\sigma_{12} B_{12}(t), \\
\ln \frac{x_{2}(t)}{x_{2}(0)}= & k_{2} t-\left(b_{2}+\alpha_{2} D_{2}\right) \int_{0}^{t} x_{2}(s) \mathrm{d} s+D_{2} \mathrm{e}^{-d_{1} \tau_{1}} \int_{0}^{t} x_{1}\left(s-\tau_{1}\right) \mathrm{d} s \\
& +\sigma_{21} B_{21}(t)-\sigma_{22} B_{22}(t) \\
= & k_{2} t-\left(b_{2}+\alpha_{2} D_{2}\right) \int_{0}^{t} x_{2}(s) \mathrm{d} s+D_{2} \mathrm{e}^{-d_{1} \tau_{1}} \int_{0}^{t} x_{1}(s) \mathrm{d} s \\
& +D_{2} \mathrm{e}^{-d_{1} \tau_{1}}\left[\int_{-\tau_{1}}^{0} x_{1}(s) \mathrm{d} s-\int_{t-\tau_{1}}^{t} x_{1}(s) \mathrm{d} s\right]+\sigma_{21} B_{21}(t)-\sigma_{22} B_{22}(t) .
\end{aligned}
$$


Dividing both sides of (25) and (26) by $t$, we get

$$
\begin{aligned}
t^{-1} \ln \frac{x_{1}(t)}{x_{1}(0)}= & k_{1}-\left(b_{1}+\alpha_{1} D_{1}\right)\left\langle x_{1}\right\rangle+D_{1} \mathrm{e}^{-d_{2} \tau_{2}}\left\langle x_{2}\right\rangle \\
& +D_{1} \mathrm{e}^{-d_{2} \tau_{2}} t^{-1}\left[\int_{-\tau_{2}}^{0} x_{2}(s) \mathrm{d} s-\int_{t-\tau_{2}}^{t} x_{2}(s) \mathrm{d} s\right] \\
& +t^{-1}\left(\sigma_{11} B_{11}(t)-\sigma_{12} B_{12}(t)\right) \\
t^{-1} \ln \frac{x_{2}(t)}{x_{2}(0)}= & k_{2}-\left(b_{2}+\alpha_{2} D_{2}\right)\left\langle x_{2}\right\rangle+D_{2} \mathrm{e}^{-d_{1} \tau_{1}}\left\langle x_{1}\right\rangle \\
& +D_{2} \mathrm{e}^{-d_{1} \tau_{1}} t^{-1}\left[\int_{-\tau_{1}}^{0} x_{1}(s) \mathrm{d} s-\int_{t-\tau_{1}}^{t} x_{1}(s) \mathrm{d} s\right] \\
& +t^{-1}\left(\sigma_{21} B_{21}(t)-\sigma_{22} B_{22}(t)\right)
\end{aligned}
$$

Computing $(27) \times\left(b_{2}+\alpha_{2} D_{2}\right)+(28) \times D_{1} \mathrm{e}^{-d_{2} \tau_{2}}$ results in

$$
\begin{aligned}
\left(b_{2}+\right. & \left.\alpha_{2} D_{2}\right) t^{-1} \ln \frac{x_{1}(t)}{x_{1}(0)}+D_{1} \mathrm{e}^{-d_{2} \tau_{2}} t^{-1} \ln \frac{x_{2}(t)}{x_{2}(0)} \\
= & M_{1}-M\left\langle x_{1}\right\rangle+D_{1} \mathrm{e}^{-d_{2} \tau_{2}}\left(b_{2}+\alpha_{2} D_{2}\right) t^{-1}\left[\int_{-\tau_{2}}^{0} x_{2}(s) \mathrm{d} s-\int_{t-\tau_{2}}^{t} x_{2}(s) \mathrm{d} s\right] \\
& +D_{1} D_{2} \mathrm{e}^{-\left(d_{1} \tau_{1}+d_{2} \tau_{2}\right)} t^{-1}\left[\int_{-\tau_{1}}^{0} x_{1}(s) \mathrm{d} s-\int_{t-\tau_{1}}^{t} x_{1}(s) \mathrm{d} s\right] \\
& +t^{-1}\left[\left(b_{2}+\alpha_{2} D_{2}\right)\left(\sigma_{11} \mathrm{~d} B_{11}(t)-\sigma_{12} \mathrm{~d} B_{12}(t)\right)\right. \\
& \left.+D_{1} \mathrm{e}^{-d_{2} \tau_{2}}\left(\sigma_{21} \mathrm{~d} B_{21}(t)-\sigma_{22} \mathrm{~d} B_{22}(t)\right)\right] .
\end{aligned}
$$

Computing $(27) \times D_{2} \mathrm{e}^{-d_{1} \tau_{1}}+(28) \times\left(b_{1}+\alpha_{1} D_{1}\right)$ leads to

$$
\begin{aligned}
D_{2} \mathrm{e}^{-d_{1} \tau_{1}} t^{-1} \ln \frac{x_{1}(t)}{x_{1}(0)}+\left(b_{1}+\alpha_{1} D_{1}\right) t^{-1} \ln \frac{x_{2}(t)}{x_{2}(0)} \\
=M_{2}-M\left\langle x_{2}\right\rangle+D_{1} D_{2} \mathrm{e}^{-\left(d_{1} \tau_{1}+d_{2} \tau_{2}\right)} t^{-1}\left[\int_{-\tau_{2}}^{0} x_{2}(s) \mathrm{d} s-\int_{t-\tau_{2}}^{t} x_{2}(s) \mathrm{d} s\right] \\
\quad+\left(b_{1}+\alpha_{1} D_{1}\right) D_{2} \mathrm{e}^{-d_{1} \tau_{1}} t^{-1}\left[\int_{-\tau_{1}}^{0} x_{1}(s) \mathrm{d} s-\int_{t-\tau_{1}}^{t} x_{1}(s) \mathrm{d} s\right] \\
\quad+t^{-1}\left[D_{2} \mathrm{e}^{-d_{1} \tau_{1}}\left(\sigma_{11} \mathrm{~d} B_{11}(t)-\sigma_{12} \mathrm{~d} B_{12}(t)\right)\right. \\
\left.+\left(b_{1}+\alpha_{1} D_{1}\right)\left(\sigma_{21} \mathrm{~d} B_{21}(t)-\sigma_{22} \mathrm{~d} B_{22}(t)\right)\right] .
\end{aligned}
$$

From the property of limit superior, for sufficiently large $t$, we can get the following equations from (27) and (28):

$$
\begin{aligned}
t^{-1} \ln x_{1}(t) \leq & k_{1}+\epsilon_{1}-\left(b_{1}+\alpha_{1} D_{1}\right)\left\langle x_{1}\right\rangle+D_{1} \mathrm{e}^{-d_{2} \tau_{2}}\left\langle x_{2}\right\rangle^{*} \\
& +t^{-1} \sigma_{11} B_{11}(t)-t^{-1} \sigma_{12} B_{12}(t), \\
t^{-1} \ln x_{2}(t) \leq & k_{2}+\epsilon_{2}-\left(b_{2}+\alpha_{2} D_{2}\right)\left\langle x_{2}\right\rangle+D_{2} \mathrm{e}^{-d_{1} \tau_{1}}\left\langle x_{1}\right\rangle^{*} \\
& +t^{-1} \sigma_{21} B_{21}(t)-t^{-1} \sigma_{22} B_{22}(t) .
\end{aligned}
$$


Let $\beta_{1}=k_{1}+\epsilon_{1}+D_{1} \mathrm{e}^{-d_{2} \tau_{2}}\left\langle x_{2}\right\rangle^{*}, \beta_{2}=k_{2}+\epsilon_{2}+D_{2} \mathrm{e}^{-d_{1} \tau_{1}}\left\langle x_{1}\right\rangle^{*}$. So (31) and (32) can be rewritten as

$$
\begin{aligned}
& t^{-1} \ln x_{1}(t) \leq \beta_{1}-\left(b_{1}+\alpha_{1} D_{1}\right)\left\langle x_{1}\right\rangle+t^{-1} \sigma_{11} B_{11}(t)-t^{-1} \sigma_{12} B_{12}(t), \\
& t^{-1} \ln x_{2}(t) \leq \beta_{2}-\left(b_{2}+\alpha_{2} D_{2}\right)\left\langle x_{2}\right\rangle+t^{-1} \sigma_{21} B_{21}(t)-t^{-1} \sigma_{22} B_{22}(t) .
\end{aligned}
$$

To prove conclusion (i), we suppose that $\left\langle x_{1}\right\rangle^{*}>0$. If $\omega \in\left\{\left\langle x_{2}(t, \omega)\right\rangle^{*}>0\right\}$, then applying Lemma 2.1 to (34) results in

$$
\left\langle x_{2}(t, \omega)\right\rangle^{*} \leq \frac{\beta_{2}}{b_{2}+\alpha_{2} D_{2}}=\frac{k_{2}+\epsilon_{2}+D_{2} \mathrm{e}^{-d_{1} \tau_{1}}\left\langle x_{1}(t)\right\rangle^{*}}{b_{2}+\alpha_{2} D_{2}} .
$$

For sufficiently large $t$, substituting (21) and (23) into (30) yields

$$
M\left\langle x_{2}(t, \omega)\right\rangle^{*} \leq M_{2}+\epsilon
$$

Since $M>0$, the left side of the last inequality is positive. Letting $\epsilon$ be small enough, we would get $M_{2} \geq 0$. Actually, since $k_{i}<0(i=1,2)$, we obtain $M_{2}<0$. This is a contradiction. Hence, $\mathcal{P}\left\{\left\langle x_{2}(t, \omega)\right\rangle^{*}>0\right\}=0$, so $\left\langle x_{2}(t)\right\rangle^{*}=0$ a.s. Then, using it in (31) and noting that $k_{1}<0$, we get that $\lim _{t \rightarrow+\infty} x_{1}(t)=0$ a.s., which is contradicts the supposition $\left\langle x_{1}\right\rangle^{*}>0$. Consequently, we have

$$
\left\langle x_{1}\right\rangle^{*}=0 \quad \text { a.s. }
$$

We are in the position to prove that $\lim _{t \rightarrow+\infty} x_{2}(t)=0$ a.s. Since $\left\langle x_{1}\right\rangle^{*}=0$ a.s., for sufficiently large $t$, we can derive from (32) that

$$
t^{-1} \ln x_{2}(t) \leq k_{2}+\epsilon_{2}-\left(b_{2}+\alpha_{2} D_{2}\right)\left\langle x_{2}\right\rangle+t^{-1} \sigma_{21} B_{21}(t)-t^{-1} \sigma_{22} B_{22}(t)
$$

Since $k_{2}<0$, applying Lemma 2.1 to the last inequality, we get $\lim _{t \rightarrow+\infty} x_{2}(t)=0$ a.s.

Now we are ready to prove (ii). Since $k_{1}>0$ and $M_{2}<0$, by Lemma 2.3 we have $\lim _{t \rightarrow+\infty} y_{2}(t)=0$ a.s. Consequently, by $(20), \lim _{t \rightarrow+\infty} x_{2}(t)=0$ a.s. Then model (4) is simplified as the following single-species model:

$$
\mathrm{d} x_{1}(t)=x_{1}(t)\left[r_{1}-E_{1}-\left(b_{1}+\alpha_{1} D_{1}\right) x_{1}(t)\right] \mathrm{d} t+\sigma_{11} x_{1}(t) \mathrm{d} B_{11}(t)-\sigma_{12} x_{1}(t) \mathrm{d} B_{12}(t)
$$

which coincides with the first equation in (5). Then applying Lemma 2.3 to the last equation leads to

$$
\lim _{t \rightarrow+\infty}\left\langle x_{1}\right\rangle=\frac{k_{1}}{b_{1}+\alpha_{1} D_{1}} \quad \text { a.s. }
$$

Using Lemma 2.4 and (15), then we can prove (iii), and the proof is similar to that of (ii). So the details are omitted.

Let us prove (iv). Substituting (22), (23), and (24) into (29) yields

$$
\begin{aligned}
\left(b_{2}+\right. & \left.\alpha_{2} D_{2}\right) t^{-1} \ln \frac{x_{1}(t)}{x_{1}(0)} \\
\geq & M_{1}-\epsilon^{\prime}-M\left\langle x_{1}\right\rangle+t^{-1}\left[\left(b_{2}+\alpha_{2} D_{2}\right)\left(\sigma_{11} \mathrm{~d} B_{11}(t)-\sigma_{12} \mathrm{~d} B_{12}(t)\right)\right. \\
& \left.+D_{1} \mathrm{e}^{-d_{2} \tau_{2}}\left(\sigma_{21} \mathrm{~d} B_{21}(t)-\sigma_{22} \mathrm{~d} B_{22}(t)\right)\right] .
\end{aligned}
$$


Noting that $M_{1}>0$, let $\epsilon^{\prime}$ be sufficiently small such that $M_{1}-\epsilon^{\prime}>0$. Consequently, $\left\langle x_{1}\right\rangle_{*} \geq$ $\frac{M_{1}-\epsilon^{\prime}}{M}$. By the arbitrariness of $\epsilon^{\prime}$ we can observe that

$$
\left\langle x_{1}\right\rangle_{*} \geq \frac{M_{1}}{M} \quad \text { a.s. }
$$

It is not hard to find that $\left\langle x_{1}\right\rangle_{*}>0$. Hence, $\beta_{1}>0$. Otherwise, it is easy to see that $\left\langle x_{1}\right\rangle_{*}=0$ from inequality (33). Similarly, by using (20), (22), and (24) to (30), we have

$$
\begin{aligned}
\left(b_{1}+\right. & \left.\alpha_{1} D_{1}\right) t^{-1} \ln \frac{x_{2}(t)}{x_{2}(0)} \\
\geq & M_{2}-\epsilon^{\prime}-M\left\langle x_{2}\right\rangle+t^{-1}\left[D_{2} \mathrm{e}^{-d_{1} \tau_{1}}\left(\sigma_{11} \mathrm{~d} B_{11}(t)-\sigma_{12} \mathrm{~d} B_{12}(t)\right)\right. \\
& \left.+\left(b_{1}+\alpha_{1} D_{1}\right)\left(\sigma_{21} \mathrm{~d} B_{21}(t)-\sigma_{22} \mathrm{~d} B_{22}(t)\right)\right] .
\end{aligned}
$$

According to Lemma 2.1, we would get

$$
\left\langle x_{2}\right\rangle_{*} \geq \frac{M_{2}}{M}>0 \quad \text { a.s. }
$$

So we have $\beta_{2}>0$. Using Lemma 2.1, from (33) and (34) we have

$$
\left\langle x_{1}\right\rangle^{*} \leq \frac{\beta_{1}}{b_{1}+\alpha_{1} D_{1}}, \quad\left\langle x_{2}\right\rangle^{*} \leq \frac{\beta_{2}}{b_{2}+\alpha_{2} D_{2}} .
$$

Consequently,

$$
\left\{\begin{array}{l}
\left(b_{1}+\alpha_{1} D_{1}\right)\left\langle x_{1}\right\rangle^{*}-D_{1} \mathrm{e}^{-d_{2} \tau_{2}}\left\langle x_{2}\right\rangle^{*} \leq k_{1}+\epsilon_{1}, \\
-D_{2} \mathrm{e}^{-d_{1} \tau_{1}}\left\langle x_{1}\right\rangle^{*}+\left(b_{2}+\alpha_{2} D_{2}\right)\left\langle x_{2}\right\rangle^{*} \leq k_{2}+\epsilon_{2}
\end{array}\right.
$$

Solving these inequalities and using the arbitrariness of $\epsilon_{i}(i=1,2)$ lead to

$$
\left\langle x_{1}\right\rangle^{*} \leq \frac{M_{1}}{M}, \quad\left\langle x_{2}\right\rangle^{*} \leq \frac{M_{2}}{M} \quad \text { a.s. }
$$

Then (iv) can be proved by combining these inequalities with (37) and (39).

The proof of Theorem 2.1 is complete.

Remark 1 Similarly to the proof of the Theorem 2.1, we would get:

(v) If $k_{1}=0, k_{2}<0$, then $x_{1}$ is nonpersistent, that is, $\lim _{t \rightarrow+\infty} t^{-1} \int_{0}^{t} x_{1}(s) \mathrm{d} s=0$, and $x_{2}$ goes to extinction;

(vi) If $k_{1}<0, k_{2}=0$, then $x_{1}$ goes to extinction, and $x_{2}$ is nonpersistent, that is, $\lim _{t \rightarrow+\infty} t^{-1} \int_{0}^{t} x_{2}(s) \mathrm{d} s=0$

(vii) If $k_{1}=0$ and $k_{2}=0$, then both $x_{1}$ and $x_{2}$ are nonpersistent.

\section{Optimal harvesting}

From Section 2 we can observe that both species $x_{1}$ and $x_{2}$ are persistent in mean if $M_{i}>0$, $i=1,2$. Our aim in this section is to gain the optimal harvesting effort such that ESY $Y(E)=$ $\lim _{t \rightarrow+\infty} \sum_{i=1}^{2} \mathbb{E}\left(E_{i} x_{i}(t)\right)$ can get the maximum when the species are persistent. We first introduce some lemmas. 
Lemma 3.1 Suppose that $x(t)$ is a solution of model (4) with any given initial value. For any $q>0$, there is a $K(q)$ such that

$$
\limsup _{t \rightarrow+\infty} \mathbb{E}|x(t)|^{q} \leq K(q)
$$

Applying Itô's formula to $\mathrm{e}^{t}\left(x_{1}^{q}+x_{2}^{q}\right)$, we would get the conclusion. It is similar to Lemma 3 of $[10]$ and is omitted it here.

Then we can prove the following lemma.

Lemma 3.2 If $b_{1}+\alpha_{1} D_{1}>D_{2} \mathrm{e}^{-d_{1} \tau_{1}}, b_{2}+\alpha_{2} D_{2}>D_{1} \mathrm{e}^{-d_{2} \tau_{2}}$, then model (4) is asymptotically stable in distribution, that is, as $t \rightarrow+\infty$, for any $\xi(t) \in C\left([-\tau, 0] ; R_{+}^{2}\right)$, there is a unique probability measure $v(\cdot)$ such that the transition probability density $p(t, \xi, \cdot)$ of $x(t)$ converges weakly to $v(\cdot)$.

Proof Let $\left(x_{1}(t ; \xi), x_{2}(t ; \xi)\right)^{T}$ and $\left(x_{1}(t ; \zeta), x_{2}(t ; \zeta)\right)^{T}$ be two solutions of model (4) with initial values $\xi(\theta) \in C\left([-\tau, 0] ; R_{+}^{2}\right)$ and $\zeta(\theta) \in C\left([-\tau, 0] ; R_{+}^{2}\right)$, respectively. Applying Itồs formula to

$$
\begin{aligned}
V(t)= & \sum_{i=1}^{2}\left|\ln x_{i}(t ; \xi)-\ln x_{i}(t ; \zeta)\right|+D_{1} \mathrm{e}^{-d_{2} \tau_{2}} \int_{t-\tau_{2}}^{t}\left|x_{2}(s ; \xi)-x_{2}(s ; \zeta)\right| \mathrm{d} s \\
& +D_{2} \mathrm{e}^{-d_{1} \tau_{1}} \int_{t-\tau_{1}}^{t}\left|x_{1}(s ; \xi)-x_{1}(s ; \zeta)\right| \mathrm{d} s
\end{aligned}
$$

leads to

$$
\begin{aligned}
\mathrm{d}^{+} V(t)= & -\sum_{i=1}^{2}\left(b_{i}+\alpha_{i} D_{i}\right)\left|x_{i}(t ; \xi)-x_{i}(t ; \zeta)\right| \mathrm{d} t \\
& +\sum_{i=1}^{2} \sum_{j=1, j \neq i}^{2} D_{i} \mathrm{e}^{-d_{j} \tau_{j}}\left|x_{j}(t ; \xi)-x_{j}(t ; \zeta)\right| \mathrm{d} t \\
& -\sum_{i=1}^{2} \sum_{j=1, j \neq i}^{2} D_{i} \mathrm{e}^{-d_{j} \tau_{j}}\left|x_{j}\left(t-\tau_{j} ; \xi\right)-x_{j}\left(t-\tau_{j} ; \zeta\right)\right| \mathrm{d} t \\
& +\sum_{i=1}^{2} \sum_{j=1, j \neq i}^{2} D_{i} \mathrm{e}^{-d_{j} \tau_{j}} \operatorname{sgn}\left(x_{i}(t ; \xi)-x_{i}(t ; \zeta)\right)\left(x_{j}\left(t-\tau_{j} ; \xi\right)-x_{j}\left(t-\tau_{j} ; \zeta\right)\right) \mathrm{d} t \\
\leq & -\sum_{i=1}^{2}\left(b_{i}+\alpha_{i} D_{i}\right)\left|x_{i}(t ; \xi)-x_{i}(t ; \zeta)\right| \mathrm{d} t \\
& +\sum_{i=1}^{2} \sum_{j=1, j \neq i}^{2} D_{i} \mathrm{e}^{-d_{j} \tau_{j}}\left|x_{j}(t ; \xi)-x_{j}(t ; \zeta)\right| \mathrm{d} t \\
& -\sum_{i=1}^{2} \sum_{j=1, j \neq i}^{2} D_{i} \mathrm{e}^{-d_{j} \tau_{j}}\left|x_{j}\left(t-\tau_{j} ; \xi\right)-x_{j}\left(t-\tau_{j} ; \zeta\right)\right| \mathrm{d} t \\
& +\sum_{i=1}^{2} \sum_{j=1, j \neq i}^{2} D_{i} \mathrm{e}^{-d_{j} \tau_{j}}\left|x_{j}\left(t-\tau_{j} ; \xi\right)-x_{j}\left(t-\tau_{j} ; \zeta\right)\right| \mathrm{d} t
\end{aligned}
$$




$$
\begin{aligned}
= & -\left(b_{1}+\alpha_{1} D_{1}-D_{2} \mathrm{e}^{-d_{1} \tau_{1}}\right)\left|x_{1}(t ; \xi)-x_{1}(t ; \zeta)\right| \mathrm{d} t \\
& -\left(b_{2}+\alpha_{2} D_{2}-D_{1} \mathrm{e}^{-d_{2} \tau_{2}}\right)\left|x_{2}(t ; \xi)-x_{2}(t ; \zeta)\right| \mathrm{d} t .
\end{aligned}
$$

Consequently,

$$
\begin{aligned}
\mathbb{E}(V(t)) \leq & V(0)-\left(b_{1}+\alpha_{1} D_{1}-D_{2} \mathrm{e}^{-d_{1} \tau_{1}}\right) \int_{0}^{t} \mathbb{E}\left|x_{1}(s ; \xi)-x_{1}(s ; \zeta)\right| \mathrm{d} s \\
& -\left(b_{2}+\alpha_{2} D_{2}-D_{1} \mathrm{e}^{-d_{2} \tau_{2}}\right) \int_{0}^{t} \mathbb{E}\left|x_{2}(s ; \xi)-x_{2}(s ; \zeta)\right| \mathrm{d} s
\end{aligned}
$$

Since $V(t) \geq 0$, it follows from the last inequality that

$$
\begin{aligned}
& \left(b_{1}+\alpha_{1} D_{1}-D_{2} \mathrm{e}^{-d_{1} \tau_{1}}\right) \int_{0}^{t} \mathbb{E}\left|x_{1}(s ; \xi)-x_{1}(s ; \zeta)\right| \mathrm{d} s \\
& \quad+\left(b_{2}+\alpha_{2} D_{2}-D_{1} \mathrm{e}^{-d_{2} \tau_{2}}\right) \int_{0}^{t} \mathbb{E}\left|x_{2}(s ; \xi)-x_{2}(s ; \zeta)\right| \mathrm{d} s \leq V(0)<\infty
\end{aligned}
$$

In other words, $\mathbb{E}\left|x_{i}(t ; \xi)-x_{i}(t ; \zeta)\right| \in L^{1}[0,+\infty), i=1,2$.

Moreover, from the first equation of model (4) we have

$$
\begin{aligned}
\mathbb{E}\left(x_{1}(t)\right)= & x_{1}(0)+\int_{0}^{t}\left[\left(r_{1}-E_{1}\right) \mathbb{E}\left(x_{1}(s)\right)-\left(b_{1}+\alpha_{1} D_{1}\right) \mathbb{E}\left(x_{1}^{2}(s)\right)\right. \\
& \left.+D_{1} \mathrm{e}^{-d_{2} \tau_{2}} \mathbb{E}\left(x_{1}(s) x_{2}\left(s-\tau_{2}\right)\right)\right] \mathrm{d} s .
\end{aligned}
$$

Hence, $\mathbb{E}\left(x_{1}(t)\right)$ is a continuously differentiable function. By Lemma 3.1 we can obtain

$$
\frac{\mathrm{d} \mathbb{E}\left(x_{1}(t)\right)}{\mathrm{d} t} \leq\left(r_{1}-E_{1}\right) \mathbb{E}\left(x_{1}(t)\right)+D_{1} \mathrm{e}^{-d_{2} \tau_{2}} \mathbb{E}\left(x_{1}(t) x_{2}\left(t-\tau_{2}\right)\right) \leq K^{*},
$$

where $K^{*}$ is a positive constant. Therefore, $\mathbb{E}\left(x_{1}(t)\right)$ is uniformly continuous. Applying the same argument to the second equation of model (4), we can obtain that $\mathbb{E}\left(x_{2}(t)\right)$ is uniformly continuous. By the conclusion of [26] we can get

$$
\lim _{t \rightarrow+\infty} \mathbb{E}\left|x_{i}(t ; \xi)-x_{i}(t ; \zeta)\right|=0 \quad \text { a.s., } i=1,2
$$

Note that $p(t, \xi, \mathrm{d} y)$ is the transition probability density of the process $x(t)$ and $P(t, \xi, A)$ denotes the probability of event $x(t ; \xi) \in A$ with the initial value $\xi(\theta) \in C\left([-\tau, 0] ; R_{+}^{2}\right)$. By Lemma 3.1 and Chebyshev's inequlity it follows from [27] that the family of $p(t, \xi, \mathrm{d} y)$ is tight, that is, for any given $\epsilon^{*}>0$, there is a compact subset $\mathcal{K} \in R_{+}^{2}$ such that $P(t, \xi, \mathcal{K}) \geq$ $1-\epsilon^{*}$.

Let $\mathcal{P}\left(C\left([-\tau, 0] ; R_{+}^{2}\right)\right)$ be the probability measures on $C\left([-\tau, 0] ; R_{+}^{2}\right)$. For any given two measures $P_{1}, P_{2} \in \mathcal{P}$, we propose the following metric:

$$
d_{\mathbb{L}}\left(P_{1}, P_{2}\right)=\sup _{g \in \mathbb{L}}\left|\int_{R_{+}^{2}} g(x) P_{1}(\mathrm{~d} x)-\int_{R_{+}^{2}} g(x) P_{2}(\mathrm{~d} x)\right|
$$

where

$$
\mathbb{L}=\left\{g: C\left([-\tau, 0] ; R_{+}^{2}\right) \rightarrow R|| g(x)-g(y)|\leq\|x-y\|,| g(\cdot) \mid \leq 1\right\} .
$$


For any $g \in \mathbb{L}$ and $t, s>0$, we get

$$
\begin{array}{rl}
\mid \mathbb{E} & g(x(t+s ; \xi))-\mathbb{E} g(x(t ; \xi)) \mid \\
\quad= & \left|\mathbb{E}\left[\mathbb{E}\left(g(x(t+s ; \xi)) \mid \mathcal{F}_{s}\right)\right]-\mathbb{E} g(x(t ; \xi))\right| \\
\quad & \left|\int_{R_{+}^{2}} \mathbb{E}(g(x(t ; \phi))) p(s, \xi, \mathrm{d} \phi)-\mathbb{E} g(x(t ; \xi))\right| \\
\quad \leq & \int_{R_{+}^{2}}|\mathbb{E}(g(x(t ; \phi)))-\mathbb{E}(g(x(t ; \xi)))| p(s, \xi, \mathrm{d} \phi) \\
\quad \leq 2 p\left(s, \xi, U_{K}^{C}\right)+\int_{U_{K}}|\mathbb{E}(g(x(t ; \phi)))-\mathbb{E}(g(x(t ; \xi)))| p(s, \xi, \mathrm{d} \phi)
\end{array}
$$

where $U_{K}=\left\{x \in R_{+}^{2}:|x| \leq K\right\}$, and $U_{K}^{C}$ is the complementary set of $U_{K}$. Since the family of $p(t, \xi, \mathrm{d} y)$ is tight, for any given $s \geq 0$, there exists a sufficiently large $K$ such that $p\left(s, \xi, U_{K}^{C}\right)<\epsilon^{*} / 4$. By (41) there exists $T>0$ such that, for $t \geq T$, we have

$$
\sup _{g \in \mathbb{L}}|\mathbb{E} g(x(t ; \phi))-\mathbb{E} g(x(t ; \xi))| \leq \frac{\epsilon^{*}}{2}
$$

Hence, it is easy to find that $|\mathbb{E} g(x(t+s ; \xi))-\mathbb{E} g(x(t ; \xi))| \leq \epsilon^{*}$. By the arbitrariness of $g$, we have

$$
\sup _{g \in \mathbb{L}}|\mathbb{E} g(x(t+s ; \xi))-\mathbb{E} g(x(t ; \xi))| \leq \epsilon^{*}
$$

that is,

$$
d_{\mathbb{L}}(p(t+s, \xi, \cdot), p(t, \xi, \cdot)) \leq \epsilon^{*}, \quad \forall t \geq T, s>0
$$

So $\{p(t, 0, \cdot): t \geq 0\}$ is Cauchy in $\mathcal{P}$ with metric $d_{\mathbb{L}}$. There is a unique $v(\cdot) \in \mathcal{P}(C([-\tau, 0]$; $\left.R_{+}^{2}\right)$ ) such that $\lim _{t \rightarrow 0} d_{\mathbb{L}}(p(t, 0, \cdot), v(\cdot))=0$. In addition, it follows from $(41)$ that

$$
\lim _{t \rightarrow 0} d_{\mathbb{L}}(p(t, \xi, \cdot), p(t, 0, \cdot))=0
$$

Thus,

$$
\lim _{t \rightarrow 0} d_{\mathbb{L}}(p(t, \xi, \cdot), v(\cdot)) \leq \lim _{t \rightarrow 0} d_{\mathbb{L}}(p(t, \xi, \cdot), p(t, 0, \cdot))+\lim _{t \rightarrow 0} d_{\mathbb{L}}(p(t, 0, \cdot), v(\cdot))=0
$$

This completes the proof of Lemma 3.2.

For simplicity, we define other notations:

$$
B=\left(\begin{array}{cc}
b_{1}+\alpha_{1} D_{1} & -D_{1} \mathrm{e}^{-d_{2} \tau_{2}} \\
-D_{2} \mathrm{e}^{-d_{1} \tau_{1}} & b_{2}+\alpha_{2} D_{2}
\end{array}\right), \quad \Lambda=\left(\lambda_{1}, \lambda_{2}\right)^{T}=\left[B\left(B^{-1}\right)^{T}+I\right]^{-1} Q
$$

where $Q=\left(r_{1}-0.5 \sigma_{11}^{2}-0.5 \sigma_{12}^{2}, r_{2}-0.5 \sigma_{21}^{2}-0.5 \sigma_{22}^{2}\right)^{T}$, and $I$ is the unit matrix. 
Theorem 3.1 Let $b_{1}+\alpha_{1} D_{1}>D_{2} \mathrm{e}^{-d_{1} \tau_{1}}, b_{2}+\alpha_{2} D_{2}>D_{1} \mathrm{e}^{-d_{2} \tau_{2}}, M>0$ and suppose that $B^{-1}+$ $\left(B^{-1}\right)^{T}$ is positive definite.

(i) If $\lambda_{i} \geq 0, i=1,2$, and $E_{i}=\lambda_{i}, i=1,2$, we have $M_{1}>0, M_{2}>0$. Then the optimal harvesting effort is $E^{*}=\Lambda=\left[B\left(B^{-1}\right)^{T}+I\right]^{-1} Q$, and the maximum of ESY is

$$
Y^{*}=\Lambda^{T} B^{-1}(Q-\Lambda)
$$

(ii) When $E_{i}=\lambda_{i}, i=1,2$, there is $M_{i} \leq 0, i=1$ or 2 , or there is $\lambda_{i}<0, i=1$ or 2 , then the optimal harvesting policy does not exist.

Proof Let $G=\left\{E=\left(E_{1}, E_{2}\right)^{T} \in R^{2} \mid M_{i}>0, E_{i}>0, i=1,2\right\}$. When (19) holds, for every $E \in G$, if the optimal harvesting effort $E^{*}$ exists, then it must belong to $G$.

We are in the position to prove (i). It is easy to note that $\Lambda \in G$, so $G$ is ont empty. By (19), for any $E \in G$, we have

$$
\lim _{t \rightarrow+\infty} t^{-1} \int_{0}^{t} E^{T} x(s) \mathrm{d} s=\sum_{i=1}^{2} E_{i} \lim _{t \rightarrow+\infty} t^{-1} \int_{0}^{t} x_{i}(s) \mathrm{d} s=E^{T} B^{-1}(Q-E) .
$$

According to Lemma 3.2, model (4) has a unique invariant measure $v(\cdot)$. By Corollary 3.4.3 in [18] we get that $v(\cdot)$ is strong mixing. Moreover, it is ergodic by Theorem 3.2.6 in [28]. Hence, it can be derived from (3.3.2) in [28] that

$$
\lim _{t \rightarrow+\infty} t^{-1} \int_{0}^{t} E^{T} x(s) \mathrm{d} s=\int_{R_{+}^{2}} E^{T} x v(\mathrm{~d} x)
$$

Let $\rho(x)$ represent the stationary probability density of model (4). Then we have

$$
Y(E)=\lim _{t \rightarrow+\infty} \sum_{i=1}^{2} \mathbb{E}\left(E_{i} x_{i}(t)\right)=\lim _{t \rightarrow+\infty} \mathbb{E}\left(E^{T} x(t)\right)=\int_{R_{+}^{2}} E^{T} x \rho(x) \mathrm{d} x
$$

Since the invariant measure of model (4) is unique, then by the one-to-one correspondence between $\rho(x)$ and its corresponding invariant measure we have

$$
\int_{R_{+}^{2}} E^{T} x \rho(x) \mathrm{d} x=\int_{R_{+}^{2}} E^{T} x v(\mathrm{~d} x)
$$

From (43)-(46) we can get

$$
Y(E)=E^{T} B^{-1}(Q-E)
$$

Note that $\Lambda=\left(\lambda_{1}, \lambda_{2}\right)^{T}$ is the unique solution of the following equation:

$$
\begin{aligned}
\frac{\mathrm{d} Y(E)}{\mathrm{d} E} & =\frac{\mathrm{d} E^{T}}{\mathrm{~d} E} B^{-1}(Q-E)+\frac{\mathrm{d}}{\mathrm{d} E}\left[(Q-E)^{T}\left(B^{-1}\right)^{T}\right] E \\
& =B^{-1} Q-\left[B^{-1}+\left(B^{-1}\right)^{T}\right] E \\
& =0 .
\end{aligned}
$$


Hence, $\Lambda=\left[B\left(B^{-1}\right)^{T}+I\right]^{-1} Q$. We can see that $\Lambda$ is the unique extreme point of $Y(E)$ because the following Hessian matrix is negative define:

$$
\begin{aligned}
\frac{\mathrm{d}}{\mathrm{d} E^{T}}\left[\frac{\mathrm{d} Y(E)}{\mathrm{d} E}\right] & =\left(\frac{\mathrm{d}}{\mathrm{d} E}\left[\left(\frac{\mathrm{d} Y(E)}{\mathrm{d} E}\right)^{T}\right]\right)^{T}=\left(\frac{\mathrm{d}}{\mathrm{d} E}\left[Q^{T}\left(B^{-1}\right)^{T}-E^{T}\left[B^{-1}+\left(B^{-1}\right)^{T}\right]\right]\right)^{T} \\
& =-B^{-1}-\left(B^{-1}\right)^{T}
\end{aligned}
$$

If $\Lambda \in G$, that is, $\lambda_{i} \geq 0(i=1,2)$ and $M_{i}>0(i=1,2)$, then $E^{*}=\Lambda$, and (41) is the maximum value of ESY.

Now we are going to prove (ii). Suppose that the optimal harvesting effort $\bar{E}^{*}=\left(\bar{E}_{1}^{*}, \bar{E}_{2}^{*}\right)^{T}$ exists. So $\bar{E}^{*} \in G$, that is, $\left.M_{i}\right|_{E_{i}=\bar{E}_{i}^{*}, i=1,2}>0, \bar{E}_{i}^{*} \geq 0, i=1$, 2 . In other words, if $\bar{E}^{*}$ is the optimal harvesting effort, then $\bar{E}^{*}$ must be the unique solution of (48). However, $\Lambda=\left(\lambda_{1}, \lambda_{2}\right)^{T}$ is also a solution of (48). Hence, $\lambda_{i}=\bar{E}_{i}^{*} \geq 0, i=1,2$, and $\left.M_{i}\right|_{E_{i}=\lambda_{i}, i=1,2}=\left.M_{i}\right|_{E_{i}=\bar{E}_{i}^{*}, i=1,2}>0$, $i=1,2$. It is a contradiction with the condition.

This completes the proof of Theorem 3.1.

\section{Numerical simulations and discussion}

Taking white noises into account, in this paper, we consider a stochastic delay model of species in two habitants. Theorem 2.1 describes sufficient conditions for persistence in the mean and extinction, which are derived from the stochastic analysis theory. Furthermore, an ergodic method is applied to show that the stochastic model has a unique stationary distribution. We obtain the optimal harvesting effort and the maximum of ESY in Theorem 3.1 by using Hessian matrix method and optimal harvesting theory of differential equations.

By using the Euler scheme in [29] to illustrate the biological significance of the results, first, it is necessary to discretize model (4). Setting the step size $\Delta t=0.01$ and the delays $\tau_{1}=2, \tau_{2}=1$, respectively, the discretized equations with respect to (4) are sa follows:

$$
\left\{\begin{aligned}
x_{1(k+1)}= & x_{1 k}+x_{1 k}\left[r_{1}-E_{1}-b_{1} x_{1 k}+D_{1} \mathrm{e}^{-d_{2} \tau_{2}} x_{2(k-100)}-\alpha_{1} D_{1} x_{1 k}\right] \Delta t \\
& +\sigma_{11} x_{1 k}\left(\triangle B_{11}\right)_{k}-\sigma_{12} x_{1 k}\left(\triangle B_{12}\right)_{k}, \\
x_{2(k+1)}= & x_{2 k}+x_{2 k}\left[r_{2}-E_{2}-b_{2} x_{2 k}+D_{2} \mathrm{e}^{-d_{1} \tau_{1}} x_{1(k-200)}-\alpha_{2} D_{2} x_{2 k}\right] \Delta t \\
& +\sigma_{21} x_{2 k}\left(\triangle B_{21}\right)_{k}-\sigma_{22} x_{2 k}\left(\triangle B_{22}\right)_{k},
\end{aligned}\right.
$$

where $\left(\triangle B_{i j}\right)_{k}=B_{i}((k+1) \triangle t)-B_{i}(k \Delta t), i, j=1,2, k=0,1,2, \ldots$. The discretization form is the approximate numerical solution related to (4). Other parameters are set as follows: $r_{1}=0.5, r_{2}=0.05, b_{1}=0.5, b_{2}=0.6, d_{1}=0.1, d_{2}=0.01, \tau_{1}=2, \tau_{2}=1, \alpha_{1}=0.4, \alpha_{2}=0.3$, with initial values $\xi_{1}(\theta)=0.3+0.03 \sin \theta$ and $\xi_{2}(\theta)=0.1+0.05 \cos \theta, \theta \in[-2,0]$.

Notice that

$$
\begin{array}{ll}
\text { (b) } k_{1}=-0.025<0, & k_{2}=-0.0013<0 ; \\
\text { (c) } k_{1}=0.0997>0, & M_{2}=-0.0151<0 ; \\
\text { (d) } M_{1}=0.1199>0, & M_{2}=0.0149>0 .
\end{array}
$$

In Figure $1(\mathrm{~b}), \sigma_{11}=0.3, \sigma_{12}=0.4, \sigma_{21}=0.15, \sigma_{22}=0.2$, the intensity is large enough such that $k_{i}<0, i=1,2$, and it causes the species extinction. In Figure $1(\mathrm{c}), \sigma_{21}=0.3, \sigma_{22}=0.3$ are large such that $M_{2}<0$, so noises affect the persistence of species $x_{2}$. When the inten- 
(a)

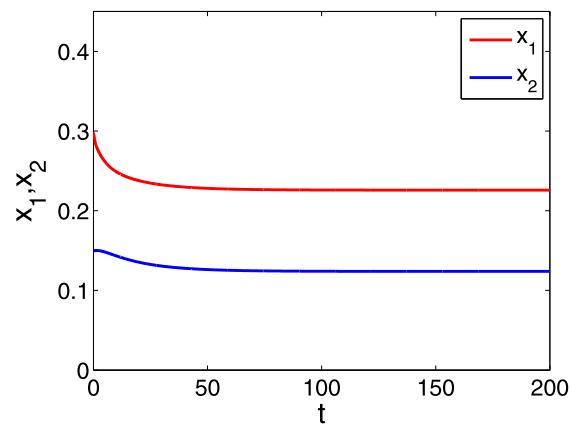

(c)

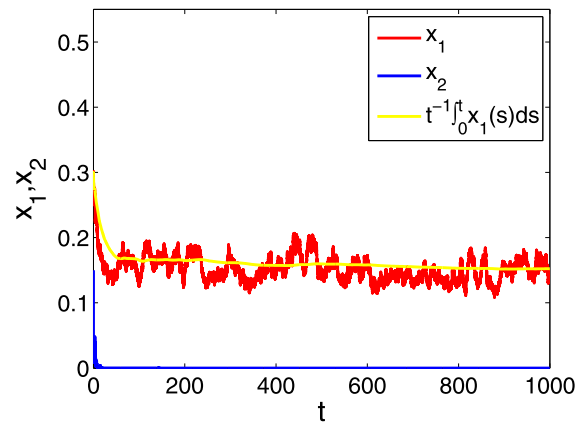

(b)

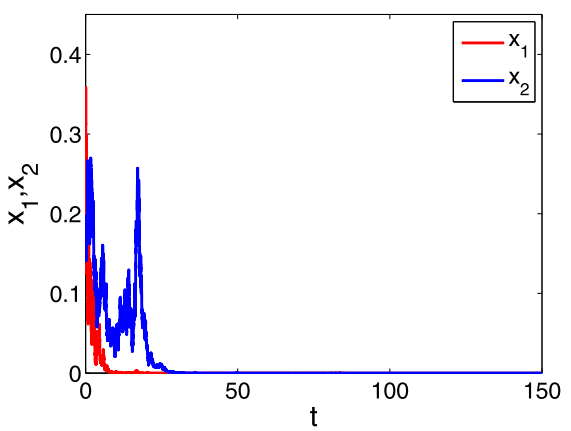

(d)

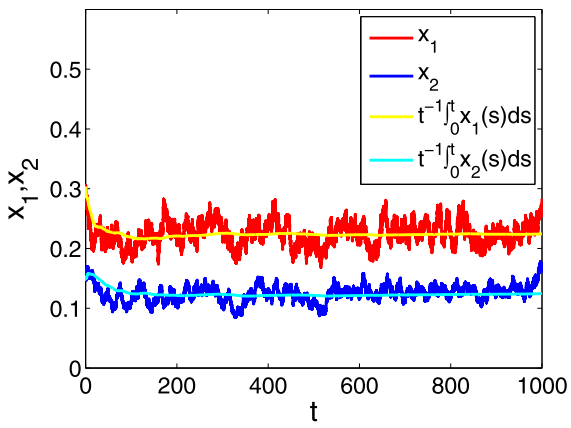

Figure 1 Simulation of the species $x_{1}(t), x_{2}(t)$ under stochastic environment. Some paraments are taken: $r_{1}=0.5, r_{2}=0.05, E_{1}=0.4, E_{2}=0.02, b_{1}=0.5, b_{2}=0.6, D_{1}=0.4, D_{2}=0.3, d_{1}=0.1, d_{2}=0.01, \tau_{1}=2$, $\tau_{2}=1 ; \alpha_{1}=0.4, \alpha_{2}=0.3, \xi_{1}(\theta)=0.3+0.03 \sin \theta, \xi_{2}(\theta)=0.1+0.05 \cos \theta, \theta \in[-2,0]$

(a) $\sigma_{11}=\sigma_{12}=\sigma_{21}=\sigma_{22} \equiv 0$; (b) $\sigma_{11}=0.3, \sigma_{12}=0.4, \sigma_{21}=0.15, \sigma_{22}=0.2$; (c) $\sigma_{11}=0.02, \sigma_{12}=0.01, \sigma_{21}=0.3$, $\sigma_{22}=0.3 ;$ (d) $\sigma_{11}=0.02, \sigma_{12}=0.01, \sigma_{21}=0.01, \sigma_{22}=0.02$.

sity of the white noise is small, the species can still be persistent just as the deterministic model; see Figure 1(d). Hence, it shows that noise with small intensity can allow the species to preserve the prosperity, whereas noise with large intensity may be a cause of species extinction.

In Figure 2(a), notice that $D_{1}=D_{2} \equiv 0$ and by computing we can obtain that $k_{1}=$ $0.0997>0, k_{2}=-0.0004<0$, and $M_{2}=-0.0002<0$; we can derive from Theorem 2.1 that $x_{1}$ is persistent in the mean and $x_{2}$ goes to extinction. On the contrary, let $D_{1}=0.4$ and $D_{2}=0.3$, which means that there exists diffusion between patches. Then species in patch 1 would move to patch 2 . We see that the parameter $k_{2}$ is still negative, but $M_{2}=0.0488$ becomes a positive constant. Therefore, $x_{2}$ turns into persistence in the mean (see Figure 2(b)). It shows that diffusion is beneficial to the persistence of population.

In Figure 3, we choose $D_{1}=0.2, D_{2}=0.3$. By computing we get $b_{1}+\alpha_{1} D_{1}=0.58>$ $D_{2} \mathrm{e}^{-d_{1} \tau_{1}}=0.2456$ and $b_{2}+\alpha_{2} D_{2}=0.69>D_{1} \mathrm{e}^{-d_{2} \tau_{2}}=0.198$. It is not hard to estimate that $B^{-1}+\left(B^{-1}\right)^{T}$ is positive definite. From $\Lambda=\left[B\left(B^{-1}\right)^{T}+I\right]^{-1} Q$ we see $\Lambda=\left(\lambda_{1}, \lambda_{2}\right)^{T}=$ $(0.2451,0.0369)^{T}$. Then we get $M_{1}=0.1783>0$ and $M_{2}=0.07>0$. By Theorem 3.1 we obtain

$$
E_{1}^{*}=\lambda_{1}=0.2451, \quad E_{2}^{*}=\lambda_{2}=0.0369 ; \quad Y^{*}=\Lambda^{T} B^{-1}(Q-\Lambda)=0.1316,
$$

whereas $E$ is different from $E^{*}$, and the ESY satisfies $Y(E)<Y^{*}$. 
(a)

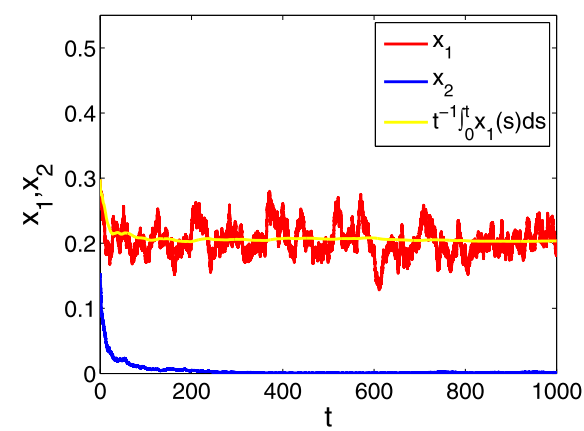

(b)

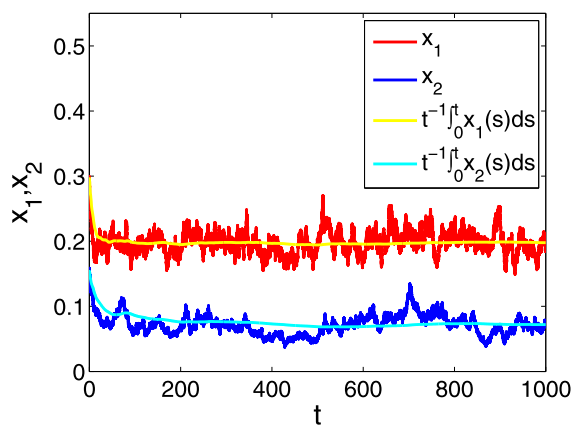

Figure 2 Simulation of the species $\boldsymbol{x}_{\mathbf{1}}(\boldsymbol{t}), \boldsymbol{x}_{\mathbf{2}}(\boldsymbol{t})$ in two habitants. Some paraments are taken: $r_{1}=0.5$, $r_{2}=0.05, E_{1}=0.4, E_{2}=0.05, b_{1}=0.5, b_{2}=0.6, d_{1}=0.1, d_{2}=0.01, \alpha_{1}=0.4, \alpha_{2}=0.3, \sigma_{11}=0.02, \sigma_{12}=0.01$, $\sigma_{21}=0.02, \sigma_{22}=0.02, \xi_{1}(\theta)=0.3+0.03 \sin \theta, \xi_{2}(\theta)=0.1+0.05 \cos \theta, \theta \in[-2,0]$. (a) $D_{1}=D_{2} \equiv 0$; (b) $D_{1}=0.4, D_{2}=0.3$.

Figure 3 The optimal harvesting effort and the maximum of ESY. Some paraments are taken: $r_{1}$ $=0.5, r_{2}=0.05, b_{1}=0.5, b_{2}=0.6, D_{1}=0.2, D_{2}=0.3$, $d_{1}=0.2, d_{2}=0.5, \alpha_{1}=0.4, \alpha_{2}=0.3, \sigma_{11}=0, \sigma_{12}=0$, $\sigma_{21}=0, \sigma_{22}=0, \xi_{1}(\theta)=0.3+0.03 \sin \theta, \xi_{2}(\theta)=0.1$ $+0.05 \cos \theta, \theta \in[-2,0]$. Red line is with $E_{1}=E_{1}^{*}$ $=0.2451, E_{2}=E_{2}^{*}=0.0369$, blue line is with $E_{1}=0.35$, $E_{2}=0.02$, and green line is with $E_{1}=0.1, E_{2}=0.2$.

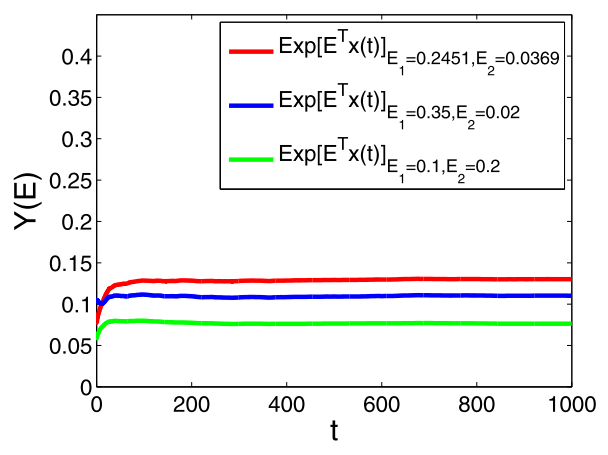

Based on theoretical analysis and numerical simulations, we present the main results in this paper:

(1) Comparing with deterministic models [8], our work extends the related results. It reveals that environment disturbance tends to have negative effects on the persistence of population. That is to say, if the intensity of noise is sufficiently large,then the species may suffer extinction, whereas the prosperity of permanence can be preserved under noise with small intensity.

(2) The Fokker-Planck equation is a classical method to handle stochastic optimal harvesting policy [25]. In this paper, we adopt a new approach, namely ergodic theory, to deal with the optimal harvesting problem, which can avoid solving the corresponding Fokker-Planck equation.

(3) Most of the existing works $[14,16,23]$ considered the effects of white noise on the growth rate, whereas we have studied not only environment disturbance on that but also harvesting effort affected by human and social factors.

The research results of this paper provide theoretic reference for some modern fields, such as fishery management. It is beneficial for people to make a rational exploitation and derive maximum profit. Some interesting topics in this direction deserve further development. We can consider diffusion coefficients disturbed by white noises or extend the present work into generalized forms, namely a multidimensional stochastic model. An- 
other interesting problem is that we can consider a more realistic but complicated model under a stochastic environment with Lévy jumps or Markovian switching.

\section{Competing interests}

The authors declare that they have no competing interests.

\section{Authors' contributions}

Both authors contributed equally to the writing of this paper. Both authors read and approved the final manuscript.

\section{Acknowledgements}

The authors would like to thank the anonymous reviewers and the editor for their valuable comments and suggestions that helped to improve the manuscript. The authors would like to thank Tonghua Zhang, who helped them during the writing of this paper. This work was supported by National Natural Science Foundation of China $(11371230,11501331)$ the SDUST Research Fund (2014TDJH102), Shandong Provincial Natural Science Foundation, China (ZR2015AQ001, BS2015SF002), Joint Innovative Center for Safe And Effective Mining Technology and Equipment of Coal Resources, Shandong Province, znd SDUST Innovation Fund for Graduate Students (No. SDKDYC170225).

\section{Received: 19 January 2016 Accepted: 4 January 2017 Published online: 17 January 2017}

\section{References}

1. Beretta, E, Takeuchi, Y: Global stability of single-species diffusion Volterra models with continuous time delays. Bull. Math. Biol. 49(4), 431-448 (1987)

2. Lu, Z, Takeuchi, Y: Global asymptotic behavior in single-species discrete diffusion systems. J. Math. Biol. 32(1), 67-77 (1993)

3. Cui, J, Takeuchi, Y, Lin, Z: Permanence and extinction for dispersal population systems. J. Math. Anal. Appl. 298(1), 73-93 (2004)

4. Meng, X, Jiao, J, Chen, L: Global dynamics behaviors for a nonautonomous Lotka-Volterra almost periodic dispersal system with delays. Nonlinear Analysis: Theory, Methods and Applications 68(12), 3633-3645 (2008)

5. Wang, Y, Xiao, Y: An epidemic model on the dispersal networks at population and individual levels. Jpn. J. Ind. Appl. Math. 32(3), 641-659 (2015)

6. Li, D, Cui, J, Song, G: Permanence and extinction for a single-species system with jump-diffusion. J. Math. Anal. Appl. 430(1), 438-464 (2015)

7. Xie, Y, Yuan, Z, Wang, L: Dynamic analysis of pest control model with population dispersal in two patches and impulsive effect. J. Comput. Sci. 5(5), 685-695 (2014)

8. Allen, LJS: Persistence and extinction in single-spices reaction-diffusion models. Bull. Math. Biol. 45(2), $209-227$ (1983)

9. Bahar, A, Mao, X: Stochastic delay population dynamics. Int. J. Pure Appl. Math. 11(4), 377-399 (2004)

10. Ji, C, Jiang, D, Shi, N: Analysis of a predator-prey model with modified Leslie-Gower and Holling-type II schemes with stochastic perturbation. J. Math. Anal. Appl. 359(2), 482-498 (2009)

11. Zhu, Q, Cao, J: Exponential stability analysis of stochastic reaction-diffusion Cohen-Grossberg neural networks with mixed delays. Neurocomputing 74(17), 3084-3091 (2011)

12. Meng, X, Zhao, S, Feng, T, Zhang, T: Dynamics of a novel nonlinear stochastic SIS epidemic model with double epidemic hypothesis. J. Math. Anal. Appl. 433, 227-242 (2016)

13. Zhu, L, Hu, H: A stochastic SIR epidemic model with density dependent birth rate. Adv. Differ. Equ. 2015(1), 1 (2015)

14. Liu, M, Qiu, H, Wang, K: A remark on a stochastic predator-prey system with time delays. Appl. Math. Lett. 26(3), 318-323 (2013)

15. Zhao, Y, Zhang, Q, Jiang, D: The asymptotic behavior of a stochastic SIS epidemic model with vaccination. Adv. Differ. Equ. 2015(1), 1 (2015)

16. Liu, M, Bai, CZ: A remark on a stochastic logistic model with diffusion. Appl. Math. Lett. 228, 141-146 (2014)

17. Zhu, Q, Cao, J, Rakkiyappan, R: Exponential input-to-state stability of stochastic Cohen-Grossberg neural networks with mixed delays. Nonlinear Dyn. 79(2), 1085-1098 (2015)

18. Meng, X, Wang, L, Zhang, T: Global dynamics analysis of a nonlinear impulsive stochastic chemostat system in a polluted environment. J. Appl. Anal. Comput. 6(3), 865-875 (2016)

19. Xia, J, Liu, Z, Yuan, R, Ruan, S: The effects of harvesting and time delay on predator-prey systems with Holling type II functional response. J. Soc. Ind. Appl. Math. 70(4), 1178-1200 (2009)

20. Zeng, G, Wang, F, Nieto, JJ: Complexity of a delayed predator-prey model with impulsive harvesting and Holling type II functional response. Adv. Complex Syst. 11(1), 77-97 (2008)

21. Song, Q, Stockbridge, RH, Zhu, C: On optimal harvesting problems in random environments. J. Soc. Ind. Appl. Math 49(2), 859-889 (2011)

22. Song, X, Chen, L: Optimal harvesting and stability for a two-species competitive system with stage structure. Math. Biosci. 170(2), 173-186 (2001)

23. Zou, X, Li, W, Wang, K: Ergodic method on optimal harvesting for a stochastic Gompertz-type diffusion process. Appl. Math. Lett. 26(1), 170-174 (2013)

24. Sharma, S, Samanta, GP: Optimal harvesting of a two species competition model with imprecise biological parameters. Nonlinear Dyn. 77(4), 1101-1119(2014)

25. Li, W, Wang, K: Optimal harvesting policy for general stochastic logistic population model. J. Math. Anal. Appl. 368(2), 420-428 (2010)

26. Barbalat, I: Systèmes d'équations différentielles d'oscillations non linéaires. Rev. Math. Pures Appl. 4, $267-270$ (1959)

27. Bao, J, Hou, Z, Yuan, C: Stability in distribution of neutral stochastic differential delay equations with Markovian switching. Stat. Probab. Lett. 79(15), 1663-1673 (2009)

28. Da Prato, G, Zabczyk, J: Ergodicity for Infinite Dimensional Systems. Cambridge University Press, Cambridge (1996)

29. Kloeden, P, Platen, E: Numerical Solution of Stochastic Differential Equations. Springer, Berlin (1992) 